\title{
Effective ecological half-lives of Cs-137 for fishes controlled by their surrounding sea-waters
}

\author{
T. Morita and K. Yoshida \\ National Research Institute of Fisheries Science, Marine Productivity Division, \\ 234-0051 Yokohama, Japan
}

\begin{abstract}
National Research Institute of Fisheries Science (NRIFS) has carried out the long term monitoring program for radioactive pollution in marine organisms caught around Japan in order to confirm the safety of marine organisms as food source. Recently, the effective ecological half-lives have been introduced to estimate the recovery time from radioactive pollution. In this study, the effective ecological half-lives of Cs-137 for some fishes were calculated from our monitoring data. It is known that fish species have each effective ecological half-lives. However, it has been unclear what change these half-lives of Cs-137 for fishes. Fishes intake Cs-137 through food chain and directly from their surrounding seawater. Therefore, the effective ecological half-lives of Cs-137 for some fishes would be controlled by the effective environment half-lives of Cs-137 for their surrounding seawater. There is difference in the effective environment half-lives between the open ocean and the coastal seawaters. Some fishes move between the open ocean and the coastal areas, and therefore their effective ecological half-lives are influenced by the effective environment half-lives for seawaters of both areas. Consequently, the rate of the influence by seawater in each area would produce the differences in the effective ecological half-lives among fish species.
\end{abstract}

\section{INTRODUCTION}

Radioactive contamination is a serious problem for the human race. To estimate the recovery time in radioactive contaminated area is very important at the view of radiation protection. Recently, the concept of the effective ecological half-lives has been introduced to estimate the recovery time and been applicable to various ecosystems [1-3]. The effective ecological half-lives describes the decrease in contaminant concentration in ecosystem over time. The effective ecological half-lives takes into account loss by physical decay and removal mechanisms which reduce the radionuclides from contaminated area by physical, chemical and biological processes. Therefore, The effective ecological half-lives is specific value to radionuclides and environment into which those are released.

The Cs-137, one of the anthropogenic gamma emitting radionuclides, is the relatively high radiotoxicity and long-term physical half-life (about $30.1 \mathrm{y}$ ), and tends to accumulate in the muscles [4]. These characters have made the Cs-137 important indicator of the radionuclide pollution. The concentrations of Cs-137 in marine environments have been extensively investigated, and particularly in Japan much attention have been paid to the concentrations of Cs-137 in marine biota which are important food sources [5 - 7].

The Cs-137 released into the environment is controlled by processes with widely different time scales, short- and long-term, through a number of different ecosystem components. In short-term process, Cs-137 rapidly removed from surface seawater by zooplankton grazing or adsorption to sedimentary particles [8]. The mechanism of long-term process has been unknown well in marine environment. The effective environment half-lives for seawater in the long-term process reported to be a time scale of year [9]. On the other hand, the removal rate of Cs-137 from fish component would firstly depend on the rate of individual fish's uptaking and eliminating Cs-137, which is a time scale 
of week-month [3]. The difference in time scale of removal rate between fish and seawater components suggests that the concentration of Cs-137 in fish component would be equilibrium with respect to the concentration in seawater component [3]. Accordingly, the effective ecological half-lives for fish component would be considered to agree with the effective environment half-lives for seawater component. However, there has been no report to show such a agreement yet.

In this study, we estimated the effective ecological or environment half-lives from the data previously reported. Our result suggested that the determinant of the effective ecological half-lives was the effective environment half-lives. We also demonstrated the agreement between the effective ecological or environment half-lives for fish and seawater components.

\section{MATERIALS AND METHODS}

\subsection{Cs-137 data set}

The Cs-137 data of fish samples are gleaned from our previous reports [10]. Fish species used in this study are below. Sampling sites are shown in Fig. 1a.

Cs-137 data of open ocean seawater are gleaned from the HAM database [11]. Data for this study are selected by following procedure: areas of the Kuroshio Current, depth $>100 \mathrm{~m}$, and sampling year (1978 -2002, expect for 1986 influenced by Chernobyl reactor accident). Sampling sites are shown in Fig. 1b.

The Cs-137 data of coastal area are gleaned from the previous monitoring reports [12]. Data for this study are selected by following procedure: depth $(>100 \mathrm{~m})$, sampling year $(1984-2002$, expect for 1986 influenced by Chernobyl reactor accident). Sampling sites are shown in Fig. 1b.
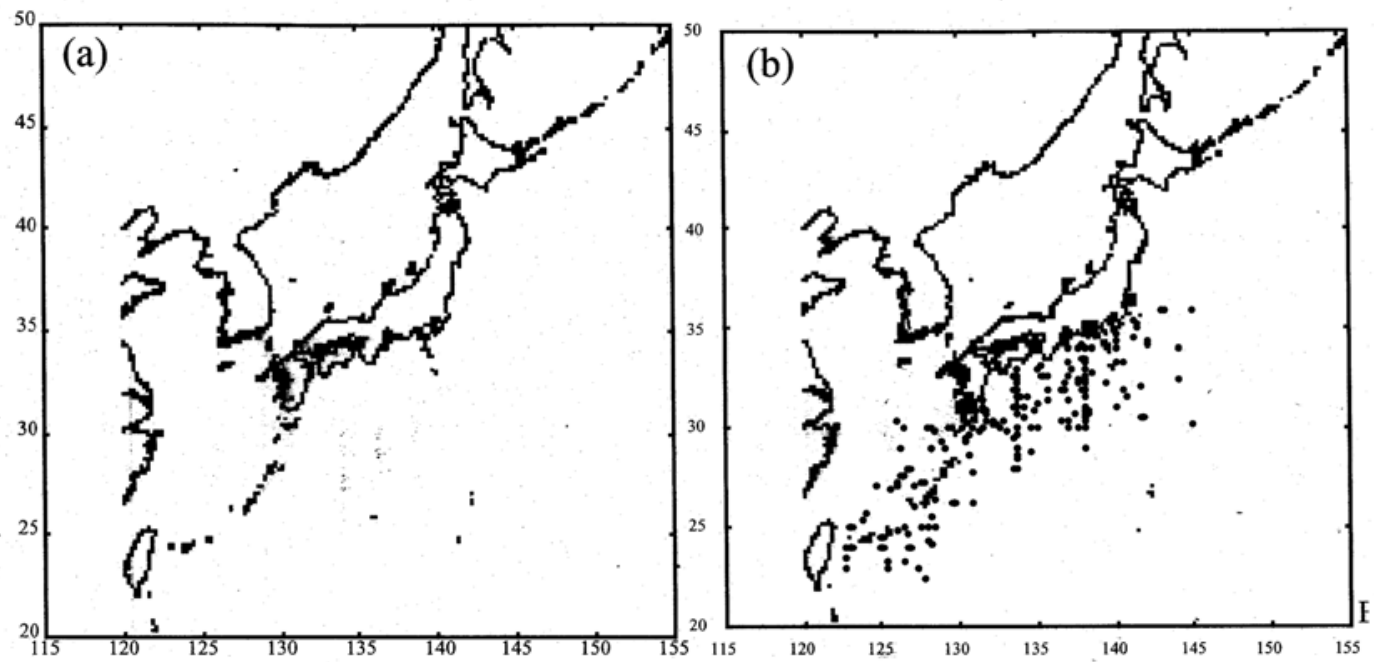

Figure 1. Map of the sampling sites of fish (a) and seawater (b). The open circles and the closed squares show the sampling sites of the open ocean seawater and the coastal seawater, respectively.

\subsection{Sample fish information}

\section{- White-spotted spinefoot, Siganus canaliculatus}

This species occur within the vicinity of river mouths especially around seagrass beds. They also occur several kilometers offshore in deep, clear waters. This species are herbivorous and eat benthic algae and seagrass. Sampling location was at the site A shown in Fig. 1a. 
- Blue-barred parrotfish, Scarus ghobban

This species inhabit lagoon and seaward reefs in slopes and drop-offs. They eat algae from rocks and corals by scraping. Sampling location was at the site A shown in Fig. 1a.

- Goatfish (Yellowstripe goatfish, Mulloidichthus flavolineatus, Yellowfin goatfish, Mulloides vanicolensis)

These adult fish inhabit sandy soil in coral reef. These adult fish are often found solitary on sand slopes. These species eat crustaceans, mollusks, worms, heart urchins and foraminiferans. Sampling location was at the site A shown in Fig. 1a.

- Blue mackerel, Scomber australasicus

This species, which occur in coastal waters and sometimes in oceanic waters, are epipelagic neritic fish forming large school. They are plankton feeders filtering copepods and other crustaceans, and sometimes feed on small fish and squids. Sampling location was at the site B shown in Fig. 1a.

- Japanese horse mackerel, Trachurus japonicus

This species is epipelagic fish and occur on continental waters. They are plankton feeders filtering copepods and other crustaceans. Sampling location was at the site B shown in Fig. 1a.

- Chub mackerel, Scomber japonicus

This species is are epipelagic neritic fish forming large school over the continental slope. This species eats copepods and other crustaceans, fishes and squids. Sampling location was at the site C shown in Fig. 1a.

\subsection{Data analysis}

Cs-137 data were plotted against the collection dates.

$$
\mathrm{Rt}=\mathrm{R}_{0} \exp ^{-\mathrm{at}}
$$

where Rt is concentration of Cs-137 in fish or each seawater component, Rt is each concentration at t year. Curves were fitted to the exponential function (1) using the least square regression method with the computer program. The effective ecological or environment half-lives was calculated from this exponential function. The effective ecological or environment half-lives consists of the radioactive decay (30.1 years for Cs-137) and removal decay from the comportment of marine fish in the present study.

In this study, fish are hypothesized to concentrate the Cs-137 from both the open ocean seawater and the coastal seawater. Thus the following equation is given:

$$
\mathrm{Af}=\mathrm{C}\{\mathrm{d} \text { Ao }+(1-\mathrm{d}) \mathrm{Ac}\}
$$

where Af, Ao and Ac were the concentration of Cs-137 for fish, open ocean seawater and coastal seawater, respectively. Ao and Ac were given as the exponential function (1). C and t were the concentration factor and the ratio of open ocean seawater, respectively. $\mathrm{C}$ and $\mathrm{t}$ were calculated by the least square methods with the computer program.

\section{RESULTS AND DISCUSSION}

Fig. 2 shows annual variation of the concentrations of Cs-137 in the open ocean seawater and coastal seawater. The annual variations of the concentration of Cs-137 were fitted to the equation (1). The calculated the effective environmental half-lives were showed in Table 1 . There was a slight difference in the effective environmental half-lives between the open ocean and the coastal seawater. The Cs-137 input into seawater was removed by the adsorption on plankton and their particulate detrital products [8]. Though such removal processes worked in both the open ocean and the coastal 
areas, the Cs-137 resuspended from land would input into the coastal seawater. Most of Cs-137 deposited recently on the land in Japan are the resuspended soil origin [13]. The resuspended Cs-137 would result in the longer effective environmental half-lives for the coastal seawater.

Cs-137 are transported through a number of different ecosystem components such fish and seawater components in this study and controlled by processes which operate on the different time scales. The concentration of Cs-137 in fish component composed of each fish individual would change at the same rate as the concentration of Cs-137 in the fish species. Fish intake Cs-137 through food chain and directly from sea waters. Therefore, the concentration of Cs-137 in fish considerably would depends on the concentration of Cs-137 in its surrounding seawater. Consequently, the concentration of Cs-137 in fish component would also depends on the concentration of Cs-137 in its surrounding seawater. If the time scale of changes in Cs-137 in seawater is greater than the time scale of change in fish, the concentration of Cs-137 in fish component would rapidly reach equilibrium and change at the same rate as the concentration of Cs-137 in seawater. In fact, the concentration of Cs-137 in fish changes rapidly because the time scale of change in fish is shorter and about 10-100 days. Therefore, it is expected that the same effective ecological half-lives for each fish component is calculated and the effective ecological half-lives is the same as the effective environmental half-lives for seawater. Fig. 3 shows annual variation of the concentrations of Cs-137 in goatfish. The calculated effective ecological half-lives were showed in Table 1. Unexpectedly, there were slight differences in the ecological environmental half-lives among sample fish. Those half-lives are not also the same as the effective environmental half-lives for either the open ocean or the coastal seawater. Thus, the equation (2) was given under the assumption that fish are hypothesized to concentrate the Cs-137 from both the open ocean and the coastal seawater. The results from the equation (2) were showed in Table 1 . The $\mathrm{R}^{2}$ from the equation (2) were similar to those from the equation (1). Our results suggested that fish species caught at the same sampling site concentrate Cs- 137 form both seawater components at the different ratio. Though the sampling site for three fish species, white-spotted spinefoot, blue-barred parrotfish and goatfish, is in the coastal area, our result also showed that the three species were influenced by open ocean water. In fact, the open ocean waters entry into the sampling site A. There are frequently the large variations of the concentration of Cs-137 among homogenous fish samples caught from the same sampling site. The large variations are also found in the annual data from those fish samples. Those variations would result in the movement of fish among the seawater components having the different ratio of change of Cs-137 concentration. Consequently, fish would have the various concentration of Cs-137.

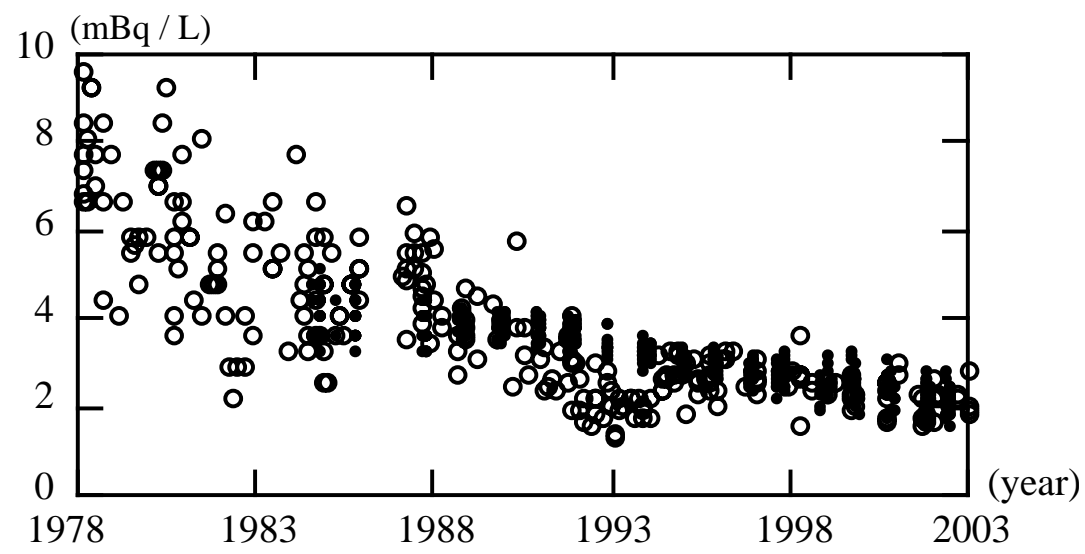

Figure 2. Annual changes of the concentration of Cs-137 in the seawater. The open and closed circles show the concentration of Cs-137 in the open ocean seawater and the coastal seawater, respectively. 


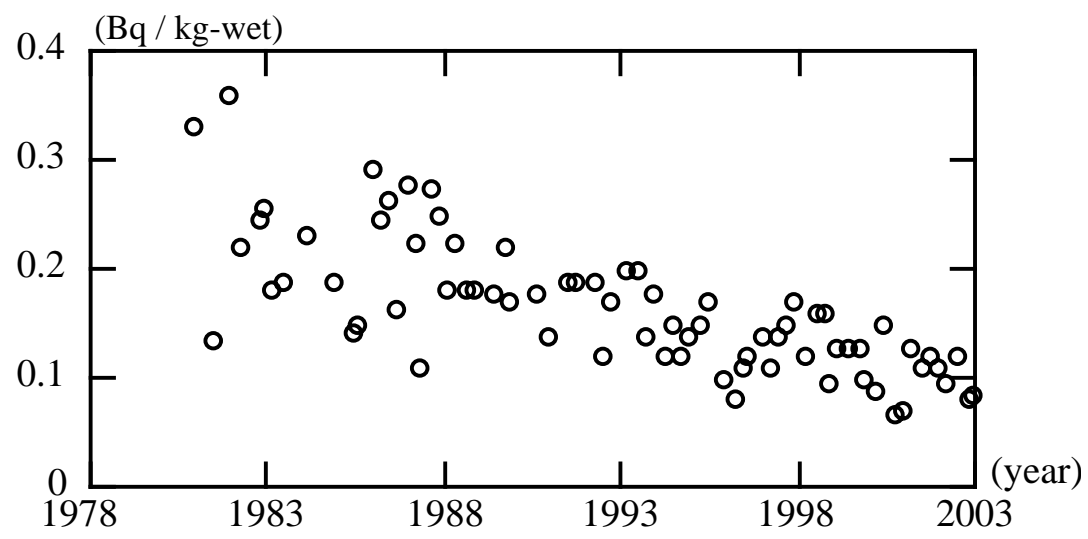

Figure 3. Annual changes of the concentration of Cs-137 in goatfish.

\section{CONCLUSION}

There is a difference in the effective environment half-lives of Cs-137 between the open ocean and the coastal seawaters because they have the different input sources of Cs-137. Some fishes move between the open ocean and the coastal areas, and therefore their effective ecological half-lives of Cs-137 are influenced by the effective environment half-lives of Cs-137 for seawater in both areas. Consequently, the rate of the influence by seawater in each area would produce the differences in the effective ecological half-lives of Cs-137 among fish species and the inconsistency of the effective ecological half-lives with the effective environmental half-lives.

Table 1. Comperison of effective ecological or environmental half lives (Teff) from the equation (1) with that from the equation (2)

\begin{tabular}{|c|c|c|c|c|}
\hline Species & Teff from the equation (1) & Teff from the equation (2) & $\mathrm{C}$ & $\mathrm{d}$ \\
\hline White-spotted spinefoot & 14.9 years $\left(R^{2}=0.60\right)$ & 14.9 years $\left(R^{2}=0.60\right)$ & 58.2 & 0.64 \\
\hline Blue-barred parrotfish & $15.7(0.48)$ & $13.6(0.48)$ & 47.4 & 0.98 \\
\hline Goatfish & $16.2(0.57)$ & $16.4(0.57)$ & 49.5 & 0.31 \\
\hline Blue mackerel & $16.9(0.63)$ & $14.4(0.64)$ & 48.5 & 0.76 \\
\hline Chub mackerel & $14.0(0.72)$ & $13.5(0.72)$ & 51.1 & 1.00 \\
\hline Japanese horse mackerel & $13.9(0.50)$ & $13.5(0.50)$ & 63.4 & 1.00 \\
\hline Open ocean seawater & $13.5(0.70)$ & - & - & - \\
\hline Coastal seawater & $18.1(0.81)$ & - & - & - \\
\hline
\end{tabular}

\section{References}

[1] Peles J. D., Bryan Jr A.L., Garten Jr C.T., Ribble D.O. and Smith M.H., Sci. Total. Environ 263 (2000) 255-262.

[2] Paller M.H., Littrell J.W. and Peters E. L. Health Phys 77 (1999) 392-402.

[3] Smith J.T., Fesenko S.V., Howard B.J., Horrill A.D., Sanzharova N.I., Alexakhin R.M., Elder D.G. and Naylor C. Environ. Sci. Technol. 33 (1999) 49-54.

[4] Young D. R. and Folsom T. R., Health Phys. 37 (1979) 703-7036. 
[5] Kasamatsu F. and Ishikawa Y., Mar. Ecol. Prog. Ser. 160 (1997) 109-120.

[6] Suzuki Y., Nakamura R. and Ueda T., Cesium-137 concentration of marine fishes from the coasts of Japan. J. Radiat. Res. 14 (1973) 382-391.

[7] Nagaya Y. and Nakamura K., Nippon Suisan Gakkaishi 53 (1987) 873-879.

[8] Fowler S.W., Ballestra S., La Rosa J., Holm E. and Lopez J.J., Nature 329 (1987) 56-58.

[9] Kasamatsu F. and Inatomi N., J. Geophys. Res. 103 (1998) 1209-1217.

[10] Annual Reports of Environmental Radioactivity Research (The Ministry of Agriculture, Forestry and Fisheries of Japan).

[11] Aoyama M. and Hirose K. ThescientificworldJOURNAL 4 (2004) 200-215.

[12] Report of Marine Radioactivity Research around Nuclear Power Stations (Ministry of Education, Culture, Sports, Science and Technology).

[13] Igarashi Y., Otsuji-Hatori M. and Hirose K., J. Environ. Radioact. 31 (1996) 157-169. 